\title{
Additivity of Entangled Channel Capacity for Quantum Input States
}

\author{
V.P.Belavkin and X.Dai \\ School of Mathematical Sciences \\ University of Nottingham, UK
}

October 15,2018

\begin{abstract}
An elementary introduction into algebraic approach to unified quantum information theory and operational approach to quantum entanglement as generalized encoding is given. After introducing compound quantum state and two types of informational divergences, namely, Araki-Umegaki (a-type) and of Belavkin-Staszewski (b-type) quantum relative entropic information, this paper treats two types of quantum mutual information via entanglement and defines two types of corresponding quantum channel capacities as the supremum via the generalized encodings. It proves the additivity property of quantum channel capacities via entanglement, which extends the earlier results of V. P. Belavkin to products of arbitrary quantum channels for quantum relative entropy of any type.
\end{abstract}

\section{Introduction}

Unlike classical channels, quantum channels can have several different capacities (e.g. for sending classical information or quantum information, one-way or two-way communication, prior or via entanglement, etc.). Well, the problem of characterizing in general the capacity of a noisy quantum channel is unsolved although several attempts have been made to define a quantum analog of Shannon mutual information (see the conceptions of coherent information [30] or von Neumann mutual entropy $[20,21,24])$. Unfortunately most of these attempts do not give a satisfactory solution because the defined quantities fail to preserve such important property of classical informational capacity 
as additivity and some do not have even monotonicity property. This paper is based on the operational entanglement approach to quantum channel capacity suggested in [10-12], which is free of the above difficulties due to the enlargement of the class of input encodings, including the encodings via entanglement for one-way communication.

Quantum entanglement is a uniquely quantum mechanical resource that plays a key role, along with the celebrating paper [28] of EinsteinPodolsky-Rosen, in many of the most interesting applications of quantum information and quantum computation, such as, Quantum entanglement is extensively used in teleporting an unknown quantum state via dual classic and Einstein-Podolsky-Rosen channels in the subject of quantum teleportation [1], quantum cryptography was investigated based on Bell's theorem [2], quantum noiseless coding theorem appeared [3] as a quantum analogous of Shannon's classical noiseless coding theorem. This paper will concentrate on application of quantum entanglement to quantum source entropy and quantum channel capacity in the subject of quantum information.

Recently tremendous effort has been made to better understand the properties of quantum entanglement as a fundamental resource of nature. Although there is as yet no complete understanding and proof of physical realizability of quantum entanglement for quantum technologies, a theoretical progress has been made in understanding this strange property of quantum mechanics, for example, mathematical aspects of quantum entanglement are extensively studied V. P. Belavkin [27] described the dynamical procedure of quantum entanglement in terms of transpose-completely positive maps in the subject of quantum decoherence and stochastic filtering theory, V. P. Belavkin and M. Ohya $[11,12]$ initiated mathematical study of quantum entanglement as truly quantum couplings from an operational view in algebraic approach, Peter Levay [15] investigated geometry of quantum entanglement for two qubits (quantum entanglement of two qubits corresponds to the twisting of the bundle), R. Penrose [16] treated quantum entanglement via spinor representation in the subject of mathematical physics, Peter Levay [17] investigated twistor geometry of quantum entanglement for three qubits still in mathematical physics. This paper will follow $[10,11,12]$ to treat with quantum entanglement in algebraic approach.

Using the operational treatment of entanglement as "true quantum" encoding V. P. Belavkin and M. Ohya [10,11,12] introduced quantum conditional entropy of the entangled compound state related to the product of marginal states which is positive and obeys all natural properties of the classical conditional entropy as the relative conditional/unconditional entropy of a compound state. They 
studied its relation to the mutual information as the informational divergence (relative informational entropy) of the compound state with respect to the product of its marginal states in the sense of Lindblad, Araki and Umegaki $[14,4,5]$. This quantum mutual information leads to an entropy bound of quantum mutual information and quantum channel capacity via entanglement (entanglement-assisted quantum capacity introduced in $[20,21]$ ), which considered the mutual information of input-output state of quantum channel. Also V. P. Belavkin and P. Staszewski [25] investigated $\mathrm{C}^{*}$-algebraic generalization of relative and conditional entropy including two types of quantum relative entropy, such as Araki-Umegaki type and Belavkin-Staszewski type, and even more general informational divergencies which meet natural axiomatic properties of relative information were studied in quantum information in [29].

Based on the combination of these two original ideas, after introducing compound quantum state and two types of quantum relative entropy, namely Araki-Umegaki type and Belavkin-Staszewski type, this paper treats two types of quantum mutual information via entanglement in algebraic approach and corresponding quantum channel capacities via entanglement in operational approach. It proves additivity property of quantum channel capacities via entanglement, which extends the results of V. P. Belavkin [10,10a] to products of arbitrary quantum channel and to quantum relative entropy of not only ArakiUmegaki type but also Belavkin-Staszewski type.

The rest of this paper is organized as follows: section two and three introduce related notion of quantum mechanics, such as quantum state and quantum entanglement respectively; section four introduces two types of quantum relative entropy via entanglement; section five introduces quantum channel capacity via entanglement and show additivity of quantum channel capacity via entanglement; final section contributes to conclusion and further problems.

\section{Quantum States in Algebraic Ap- proach}

This subsection is a brief mathematical review of Quantum State in Quantum Mechanics in a discrete algebraic approach. Anyone can turn to [6] for general physical review, or [7] for mathematical foundations of Quantum Mechanics, [8,9] for a brief review of Quantum Mechanics Principles in quantum information and computation.

In order to keep a closer link with classical information theory, we will allow for a possibility of having classical-quantum combined 
systems described in what follows by discrete non-commutative $\mathrm{W}^{*}$ algebras $\mathcal{A}=\left(\mathcal{A}_{i}\right)$ represented by block-diagonal matrices $A=\left[A(i) \delta_{j}^{i}\right]$ with arbitrary uniformly bounded operators $A(i) \in \mathcal{A}_{i}$ on some separable Hilbert spaces $\mathcal{G}_{i}$.

Let $\mathcal{H}$ denote the separable Hilbert space of a quantum system, and $\mathcal{L}(\mathcal{H})$ denote the algebra of all linear bounded operators on $\mathcal{H}$, with a decomposable subalgebra $\mathcal{B} \subseteq \mathcal{L}(\mathcal{H})$ of elements $B \in \mathcal{B}$ of the blockdiagonal form $B=\left[B(j) \delta_{j}^{i}\right]$, where $B(j) \in \mathcal{L}\left(\mathcal{H}_{j}\right)$, corresponding to an orthogonal decomposition $\mathcal{H}=\bigoplus_{j} \mathcal{H}_{j}$. Note that any such algebra is weakly closed in $\mathcal{L}(\mathcal{H})$, i.e. is a $W^{*}$-algebra having a predual space $\mathcal{B}_{*}$, which can be identified with the trace class subspace of $\mathcal{B}$ with respect to the pairing

$$
\langle\varsigma \mid B\rangle=\sum_{j} \operatorname{Tr}_{\mathcal{H}_{j}}\left[\varsigma(j)^{\dagger} B(j)\right]=\operatorname{Tr}_{\mathcal{H}}\left[B \varsigma^{\dagger}\right],
$$

where $\varsigma(j) \in \mathcal{B}_{j}$ are such operators in $\mathcal{H}_{j}$ that $\operatorname{Tr}_{\mathcal{H}} \sqrt{\varsigma^{\dagger} \varsigma}<\infty$ and $\operatorname{Tr}_{\mathcal{H}}$ is the standard trace on $\mathcal{B}$ normalized on one dimensional projectors $P_{\psi}=\psi \psi^{\dagger}$ for $\psi \in \mathcal{H}_{j}$. We now remind the definition of quantum normal state.

Definition 1 A bounded linear functional $\sigma: \mathcal{B} \rightarrow \mathbb{C}$ of the form $\sigma(B)=\operatorname{Tr}_{\mathcal{H}}[B \varsigma]$ for a $\varsigma=\varsigma^{\dagger} \in \mathcal{B}_{*}$ is called the state on $\mathcal{B}$ if it is positive for any positive operator $B \in \mathcal{B}$ and normalized $\sigma(I)=1$ for the identity operator $I$ in $\mathcal{B}$. The operator $\varsigma$, uniquely defined as a positive trace one operator on $\mathcal{H}$, is called density operator of the state $\sigma$.

Let $\mathcal{G}$ be another separable Hilbert space and $\chi$ be a HilbertSchmidt operator from $\mathcal{G}$ to $\mathcal{H}$ defining a decomposition $\varsigma=\chi \chi^{\dagger}$ of the state density with the adjoint operator $\chi^{\dagger}$ from $\mathcal{H}$ to $\mathcal{G}$. We now equip $\mathcal{G}$ with an isometric involution $J=J^{\dagger}, J^{2}=I$, the complex conjugation on $\mathcal{G}$,

$$
J \Sigma_{k} \lambda_{k} \zeta_{k}=\Sigma_{k} \overline{\lambda_{k}} J \zeta_{k}, \forall \lambda_{k} \in \mathbb{C}, \zeta_{k} \in \mathcal{G},
$$

defining an isometric transposition $\widetilde{A}=J A^{\dagger} J=\bar{A}^{\dagger}$ on the algebra $\mathcal{L}(\mathcal{G})$, where $\bar{A}=J A J$. A normal state $\rho: \mathcal{A} \rightarrow \mathbb{C}$ on the algebra $\mathcal{A} \subseteq \mathcal{L}(\mathcal{G})$ is called real (or equivalently symmetric) if its density is real, $\bar{\varrho}=\varrho$ (or equivalently symmetric, $\widetilde{\varrho}=\varrho$ ). Given a state, $J$ can be always chosen in such a way that $\varrho=\bar{\varrho}$ as it was done in $[10,11]$, but here we fix $J$ but not $\varrho$, and in general we will not assume that $\varrho=\widetilde{\varrho}$. Instead, we may assume that the transposition leaves invariant the decomposable subalgebra $\mathcal{A} \subseteq \mathcal{L}(\mathcal{G})$ such that $\overline{\mathcal{A}}:=J \mathcal{A} J=\mathcal{A}$, however from the notational and operational point of view, it is preferable to distinguish the algebra $\mathcal{A}$ from the transposed algebras $\widetilde{\mathcal{A}}=\{\widetilde{A}: A \in \mathcal{A}\}=\overline{\mathcal{A}}$. 
Lemma 1 ([10,11,12]) Any normal state $\rho$ on $\mathcal{A} \subseteq \mathcal{L}(\mathcal{G})$ can be expressed as

$$
\rho(A)=\operatorname{Tr}_{\mathcal{H}}\left[\chi \widetilde{A} \chi^{\dagger}\right]=\operatorname{Tr}_{\mathcal{H}}[A \varrho],
$$

where the density operator $\varrho \in \mathcal{A}_{*}$ is uniquely defined by $\widetilde{\varrho}=\chi^{\dagger} \chi=\bar{\varrho}$ iff $\chi^{\dagger} \chi \in \widetilde{\mathcal{A}}$.

Thus we have an operational expression $\rho(A)=\left\langle\chi \bar{A} \chi^{\dagger} \mid I\right\rangle$ of quantum normal state, which is called standard in the case $\mathcal{G}=\mathcal{H}$ and $\chi=\sqrt{\varsigma}$, in which case $\varrho=\bar{\varsigma}$. Generally $\chi$ is named as the amplitude operator, or simply amplitude given by a vector $\chi=\psi \in \mathcal{H}$ with $\psi^{\dagger} \psi=\|\psi\|^{2}=1$ in the case of one dimensional $\mathcal{G}=\mathbb{C}$, corresponding to the pure state $\sigma(B)=\psi^{\dagger} B \psi$, where $\chi^{\dagger}$ is the functional $\psi^{\dagger}$ from $\mathcal{H}$ to complex field $\mathbb{C}$.

Remark 1 The amplitude operator $\chi$ is unique up to a unitary transform in $\mathcal{H}$ as a probability amplitude satisfying the conditions $\chi^{\dagger} \chi \in \widetilde{\mathcal{A}}$ such that $\varrho=\overline{\chi^{\dagger} \chi}$ is positive decomposable trace one operator $\varrho=\oplus_{i} \varrho(i)$ with the components $\varrho(i) \in \mathcal{L}\left(\mathcal{G}_{i}\right)$ normalized as

$$
\operatorname{Tr}_{\mathcal{G}_{i}} \varrho(i)=k(i) \geq 0, \sum_{i} k(i)=1 .
$$

Therefore we can identify the predual space $\mathcal{A}_{*}$ with the direct sum $\bigoplus \mathcal{T}\left(\mathcal{G}_{i}\right) \subseteq \mathcal{A}$ of the Banach spaces $\mathcal{T}\left(\mathcal{G}_{i}\right)$ of trace class operators in $\mathcal{G}_{i}$.

Note that we denote the probability operators $P_{\mathcal{A}}=\varrho \in \mathcal{A}_{*}, P_{\mathcal{B}}=$ $\varsigma \in \mathcal{B}_{*}$ as trace densities of the states $\rho, \sigma$ defined as the expectations on the algebras $\mathcal{A}, \mathcal{B}$ respectively by the variations of Greek letters $\rho, \sigma$ which are also used in [12] for the transposed (contravariant) density operators $\widetilde{\varrho} \equiv \rho=\bar{\varrho}, \widetilde{\varsigma} \equiv \sigma=\bar{\varsigma}$ with respect to the bilinear pairings $\rho(A)=\langle A, \rho\rangle \equiv\langle\bar{\rho} \mid A\rangle, \sigma(B)=\langle B, \sigma\rangle \equiv\langle\bar{\sigma} \mid B\rangle$.

We now define an entangled state $\omega$ on the $W^{*}$-tensor product algebra $\mathcal{A} \otimes \mathcal{B}$ of bounded operators on the Hilbert product space $\mathcal{G} \otimes \mathcal{H}$ by

$$
\operatorname{Tr}_{\mathcal{G}}\left[\widetilde{A} \chi^{\dagger} B \chi\right]=\omega(A \otimes B)=\operatorname{Tr}_{\mathcal{H}}\left[\chi \widetilde{A} \chi^{\dagger} B\right] .
$$

Obviously $\omega$ can be uniquely extended by linearity to a normal state on the algebra $\mathcal{A} \otimes \mathcal{B}$ generated by all the linear combinations $C=\Sigma_{k} \lambda_{k} A_{k} \otimes B_{k}$ such that $\omega\left(C^{\dagger} C\right)=\operatorname{Tr}_{\mathcal{G}}\left[X^{\dagger} X\right] \geq 0$, where $X=$ $\Sigma_{k} \lambda_{k} B_{k} \chi \tilde{A}_{k}$, and $\omega(I \otimes I)=\operatorname{Tr}\left[\chi^{\dagger} \chi\right]=1$.

Remark 2 The state (4) is pure on $\mathcal{L}(\mathcal{G} \otimes \mathcal{H})$, since it is given by an amplitude $\psi \in \mathcal{G} \otimes \mathcal{H}$ defined as $(\zeta \otimes \eta)^{\dagger} \psi=\eta^{\dagger} \chi J \zeta, \forall \zeta \in \mathcal{G}, \eta \in \mathcal{H}$, with the states $\rho$ on $\mathcal{A}$ and $\sigma$ on $\mathcal{B}$ as the marginals of $\omega$ :

$$
\sigma(B)=\omega(I \otimes B)=\operatorname{Tr}_{\mathcal{H}}[B \varsigma], \rho(A)=\omega(A \otimes I)=\operatorname{Tr}_{\mathcal{G}}[\widetilde{A} \varrho] .
$$

Therefore, we call the state $\omega$ defined above as a pure entanglement state for $\mathcal{A}=\mathcal{L}(\mathcal{G}), \mathcal{B}=\mathcal{L}(\mathcal{H})$. 
More general, mixed entangled states for $\mathcal{A}=\mathcal{L}(\mathcal{G}), \mathcal{B}=\mathcal{L}(\mathcal{H})$ can be obtained by using a stochastic amplitude operator $\chi: \mathcal{G} \rightarrow \mathcal{F} \otimes \mathcal{H}$.

Given an amplitude operator $v: \mathcal{F} \longrightarrow \mathcal{G} \otimes \mathcal{H}$ on a Hilbert space $\mathcal{F}$ into the tensor product Hilbert space $\mathcal{G} \otimes \mathcal{H}$ such that $\varpi:=v v^{\dagger} \in$ $\mathcal{A} \otimes \mathcal{B}$ and $\operatorname{Tr}_{\mathcal{F}}\left[v^{\dagger} v\right]=1$, we define a compound state $\omega: \mathcal{A} \otimes \mathcal{B} \longrightarrow \mathbb{C}$ as

$$
\omega(A \otimes B)=\operatorname{Tr}_{\mathcal{F}}\left[v^{\dagger}(A \otimes B) v\right]=\operatorname{Tr}[(A \otimes B) \varpi] .
$$

Lemma $2([10,11,12])$ Any compound state (6) can be achieved via an entanglement $\chi$ as

$$
\operatorname{Tr}_{\mathcal{G}}\left[\widetilde{A} \chi^{\dagger}(I \otimes B) \chi\right]=\omega(A \otimes B)=\operatorname{Tr}_{\mathcal{F} \otimes \mathcal{G}}\left[\chi \widetilde{A} \chi^{\dagger}(I \otimes B)\right],
$$

with $\omega(A \otimes I)=\operatorname{Tr}_{\mathcal{G}}[A \varrho], \omega(I \otimes B)=\operatorname{Tr}_{\mathcal{H}}[B \varsigma], \widetilde{\varrho}=\chi^{\dagger} \chi$ and $\varsigma=$ $\operatorname{Tr}_{\mathcal{F}}\left[\chi \chi^{\dagger}\right]$, where $\chi$ is an operator $\mathcal{G} \longrightarrow \mathcal{F} \otimes \mathcal{H}$ with $\operatorname{Tr}_{\mathcal{F}}\left[\chi \mathcal{A} \chi^{\dagger}\right] \subset \mathcal{B}$, $\chi^{\dagger}(I \otimes B) \chi \subset \mathcal{A}$. Moreover, the operator $\chi$ is uniquely defined by $\tilde{\chi} U=v$, where

$$
(\zeta \otimes \eta)^{\dagger} \widetilde{\chi} \xi=(J \xi \otimes \eta)^{\dagger} \chi J \zeta, \quad \forall \xi \in \mathcal{F}, \zeta \in \mathcal{G}, \eta \in \mathcal{H}
$$

up to a unitary transformation $U$ of the minimal space $\mathcal{F}=\operatorname{rank} v^{\dagger}$ equipped with an isometric involution $J$. Note that we have used the invariance of trace under the transposition such that $\operatorname{Tr}_{\mathcal{G}}[\widetilde{\varrho}]=\operatorname{Tr}_{\mathcal{G}}[\varrho]$.

\section{Entanglement as Quantum Opera- tion}

Quantum entanglement is iron to the classical world's bronze age. Quantum entanglement are recently researched extensively, such as Peter Levay [15] via geometric method, Penrose [16] and Peter Levay [17] via spinor and twistor representation, Belavkin $[10,11,12]$ via algebraic approach. We now follow $[10,11,12]$ for entangled state.

Let us write the entangled state as

$$
\omega(A \otimes B)=\operatorname{Tr}_{\mathcal{H}}\left[B \pi^{*}(A)\right]=\operatorname{Tr}_{\mathcal{G}}[A \pi(B)],
$$

where the operator $\pi^{*}(A)=\operatorname{Tr}_{\mathcal{F}}\left[\chi \widetilde{A} \chi^{\dagger}\right] \in \mathcal{B}$, bounded by $\|A\| \varsigma \in \mathcal{B}_{*}$, is in the predual space $\mathcal{B}_{*}=\mathcal{T}(\mathcal{H})$ of $\mathcal{B}$ for any $A \in \mathcal{G}$, and

$$
\pi(B)=J \chi^{\dagger}\left(I \otimes B^{\dagger}\right) \chi J=\tilde{\chi}(I \otimes \widetilde{B}) \bar{\chi}
$$

with $\widetilde{B}$ defined by isometric involution in $\mathcal{H}$ as $\widetilde{B}=J B^{+} J$, is in $\mathcal{A}_{*}$ as a trace-class operator in $\mathcal{G}$, bounded by $\|B\| \varsigma \in \mathcal{A}_{*}$.

The dual linear maps $\pi$ and $\pi^{*}$ in (9), $\pi^{* *}=\pi$, with respect to the standard pairing $\langle A \mid A\rangle=\operatorname{Tr}\left[A^{*} A\right]$, are both positive, but in general 
not completely positive but transpose-completely positive maps, with $\pi^{*}(I)=\varsigma, \pi(I)=\varrho$.

Remark 3 For the entangled state $\omega(A \otimes B)=\operatorname{Tr}[(A \otimes B) \varpi]$, in terms of the compound density operator $\varpi=v v^{\dagger}$, the entanglements $\pi$ and $\pi^{*}$ can be written as

$$
\pi(B)=\operatorname{Tr}_{\mathcal{H}}[(I \otimes \widetilde{B}) \varpi], \pi^{*}(A)=\operatorname{Tr}_{\mathcal{G}}[(\widetilde{A} \otimes I) \varpi]
$$

Definition 2 ([10,11,12]) The transpose-completely positive map $\pi: \mathcal{B} \rightarrow \mathcal{A}_{*}$, (or its dual map $\pi^{*}: \mathcal{A} \rightarrow \mathcal{B}_{*}$ ), normalized as $\operatorname{Tr}_{\mathcal{G}}[\pi(I)]=$ 1 (or, equivalently, $\operatorname{Tr}_{\mathcal{H}}\left[\pi^{*}(I)\right]=1$ ) is called the quantum entanglement of the state $\sigma(B)=\operatorname{Tr}_{\mathcal{H}}[\pi(B)]$ to a state on $\mathcal{A}$ described by the density operator $\varrho=\pi(I)$ (or of $\rho(A)=\operatorname{Tr}_{\mathcal{G}}\left[\pi^{*}(A)\right]$ to $\varsigma=\pi^{*}(I)$ ).

We call the standard entanglement $\pi=\pi_{q}$ for $(\mathcal{B}, \sigma)$ the entanglement to $\varrho=\widetilde{\varsigma}$ on $\mathcal{A}=\tilde{\mathcal{B}}$ by

$$
\pi_{q}(B)=\varrho^{1 / 2} \widetilde{B} \varrho^{1 / 2}, B \in \tilde{\mathcal{B}} .
$$

Obviously $\pi_{q}^{*}(A)=\varsigma^{1 / 2} \widetilde{A} \varsigma^{1 / 2}$, where $\varsigma=\widetilde{\varrho}$, and $\pi_{q}^{*}=\pi_{q}$ iff $\mathcal{B}=\tilde{\mathcal{B}}$ and $\varsigma=\widetilde{\varsigma}$.

The standard entanglement defines the standard compound state

$$
\omega_{q}(A \otimes B)=\operatorname{Tr}_{\mathcal{H}}\left[B \varsigma^{1 / 2} \widetilde{A} \varsigma^{1 / 2}\right]=\operatorname{Tr}_{\mathcal{H}}\left[A \varrho^{1 / 2} \widetilde{B} \varrho^{1 / 2}\right] .
$$

Theorem 1 Every entanglement $\pi$ on $\mathcal{B}$ to the state $\varrho \in \mathcal{A}_{*}$ has a decomposition

$$
\pi(B)=\sqrt{\varrho \Pi(B)} \sqrt{\varrho} \equiv \pi_{q}(\Pi(B)),
$$

where $\Pi$ is a normal completely positive map $\mathcal{B} \rightarrow \tilde{\mathcal{A}}$ normalized to the identity operator at least on the minimal Hilbert subspace supporting density operator $\widetilde{\varrho}$. This decomposition is unique by the condition $\Pi(I)=E_{\widetilde{\varrho}}$, where $E_{\widetilde{\varrho}} \in \widetilde{\mathcal{A}}$ is the orthoprojector on this minimal Hilbert subspace $\tilde{\mathcal{G}}_{\varrho} \subseteq \mathcal{G}$.

Proof: $\Pi$ can be found as a solution to the linear equation

$$
\widetilde{\varrho}^{1 / 2} \Pi(B) \widetilde{\varrho}^{1 / 2} \equiv \widetilde{\pi(B)} \forall B \in \mathcal{B}
$$

which is unique if $\varrho$ and therefore $\widetilde{\varrho}$ is not degenerate:

$$
\Pi(B)=\widetilde{\varrho}^{-1 / 2} \widetilde{\pi(B)} \widetilde{\varrho}^{-1 / 2} .
$$

If $\varrho$ is degenerate, we should consider the Hilbert subspace $\mathcal{G}_{\widetilde{\varrho}}=E_{\widetilde{\varrho}} \mathcal{G}$ given by the minimal orthoprojector $E_{\widetilde{\varrho}} \in \tilde{\mathcal{A}}$ supporting the state $\widetilde{\rho}(A)=\rho(\widetilde{A})$ on the transposed algebra $\tilde{\mathcal{A}}$ such that $\widetilde{\rho}\left(E_{\widetilde{\varrho}}\right)=1$. 


\section{Quantum Mutual Information via En- tanglement}

Quantum mutual information is extensively researched in the past starting from Belavkin and Stratonovich [18] and more recently by Belavkin and Ohya [10], Belavkin and Ohya [11], Benjamin Schumacher and Michael D. Westmoreland [19]. Belavkin and Ohya [11,12] introduced quantum mutual information as the von Neumann negaentropy $\mathcal{R}(\varpi)=-\mathcal{S}(\varpi)$ of the entangled compound state related to negaentropy $\mathcal{R}(\varrho \otimes \varsigma)=-\mathcal{S}(\varrho \otimes \varsigma)$ of the product of marginal states, i.e. as the relative negaentropy $\mathcal{R}^{(\mathrm{a})}(\varpi: \varphi)=-\mathcal{S}^{(\mathrm{a})}(\varpi: \varphi)$, in the sense of Lindblad, Araki and Umegaki relative entropy [14,4,5] with respect to $\varphi=\varrho \otimes \varsigma$. Cerf and Adami [24] discussed mutual quantum information entropy and its subadditivity property via entropy diagram.

Note that we prefer to use in what is following the term "information" for negaentropy, leaving the term "entropy" for the opposite quantities like relative negainformation $\mathcal{S}^{(\mathrm{a})}(\varpi: \varphi)=-\mathcal{R}^{(\mathrm{a})}(\varpi: \varphi)$, which coincides with usual von Newmann entropy $\mathcal{S}(\varpi)$ if it is taken with respect to the trace $\phi=\operatorname{Tr}$.

We now follow $[18,11]$ to define quantum mutual information via quantum entanglement.

Definition 3 Relative quantum information of Araki-Umegaki type to compound state $\omega$ on the algebra $\mathcal{A} \otimes \mathcal{B}$, (or information divergence of the state $\omega$ with respect to a reference state $\phi$ ) is defined by the density operator $\varpi, \varphi$ of these states $\omega$ and $\phi$ as

$$
\mathcal{R}^{(\mathrm{a})}(\varpi: \varphi)=\operatorname{Tr}[\varpi(\ln \varpi-\ln \varphi)] .
$$

This quantity is used in most definitions of quantum relative information. However unlike the classical case, this is not only possible choice for informational divergence of the states $\omega$ and $\phi$, and it does not relate explicitly the informational divergence to the RadonNikodym type (RN) density $\varpi_{\phi}=\varphi^{-1 / 2} \varpi \varphi^{-1 / 2}$ of the state $\omega$ with respect to $\phi$ as in the classical case.

Another quantum relative information (of Belavkin-Staszewski type [26]) was introduced in [25] as

$$
\mathcal{R}^{(\mathrm{b})}(\varpi: \varphi)=\operatorname{Tr}\left[\varpi \ln \left(\varphi^{-1} \varpi\right)\right],
$$

where $\varpi \ln \left(\varphi^{-1} \varpi\right)=\ln \left(\varpi \varphi^{-1}\right) \varpi$ is understood as the Hermitian operator

$$
\varpi^{1 / 2} \ln \left(\varpi^{1 / 2} \varphi^{-1} \varpi^{1 / 2}\right) \varpi^{1 / 2}=v \ln \left(v^{\dagger} \varphi^{-1} v\right) v^{\dagger} .
$$


This relative information can be explicitly written in terms of the RN density $\varpi_{\phi}$ as $\mathcal{R}^{(\mathrm{b})}(\varpi: \varphi)=\phi\left(r\left(\varpi_{\phi}\right)\right)$, where $r\left(\varpi_{\phi}\right)=\varpi_{\phi} \ln \varpi_{\phi}$.

Ohya and Petz [26] were able to show that, in finite dimensions and faithful states, the Belavkin-Staszewski information divergence based on quantum relative information of Belavkin and Staszewski type gives better distinction of $\varpi$ and $\varphi$ in the sense that it is greater than relative quantum information of Araki-Umegaki type, and that it satisfies the following important property.

Lemma 3 Given a normal completely positive unital map K : $\mathcal{M} \rightarrow \mathcal{M}^{0}$, if $\omega=\omega_{0} \mathrm{~K}, \phi=\phi_{0} \mathrm{~K}$, then for both relative informations,

$$
\mathcal{R}(\varpi: \varphi) \leq \mathcal{R}\left(\varpi_{0}: \varphi_{0}\right) .
$$

Generally this is called monotonicity property of relative information, which is well known since [14,22] for Araki-Umegaki type, while it is less known that Belavkin-Staszewski type also satisfies all axioms for quantum relative entropy including this inequality. Of course it is worth mathematically proving this inequality of Belavkin-Staszewski type in the most general case.

Definition 4 We define the mutual quantum information $\mathcal{I}_{\mathcal{A}, \mathcal{B}}(\pi)=$ $\mathcal{I}_{\mathcal{B}, \mathcal{A}}\left(\pi^{*}\right)$ of both types in a compound state $\omega$ achieved by a quantum entanglement $\pi: \mathcal{B} \rightarrow \mathcal{A}_{*}$, or by $\pi^{*}: \mathcal{A} \rightarrow \mathcal{B}_{*}$ with

$$
\rho(A)=\omega(A \otimes I)=\operatorname{Tr}_{\mathcal{G}}[A \varrho], \sigma(B)=\omega(I \otimes B)=\operatorname{Tr}_{\mathcal{H}}[B \varsigma]
$$

as the relative information of each type of the state $\omega$ on $\mathcal{M}=\mathcal{A} \otimes \mathcal{B}$ with the respect to the product state $\phi=\rho \otimes \sigma$ :

$$
\begin{gathered}
\mathcal{I}_{\mathcal{A}, \mathcal{B}}^{(\mathrm{a})}(\pi)=\operatorname{Tr}[\varpi(\ln \varpi-\ln (\varrho \otimes I)-\ln (I \otimes \varsigma))] . \\
\mathcal{I}_{\mathcal{A}, \mathcal{B}}^{(\mathrm{b})}(\pi)=\operatorname{Tr}\left[\varpi \ln \left((\varrho \otimes \varsigma)^{-1} \varpi\right)\right] .
\end{gathered}
$$

The definition of mutual quantum entropy for Araki-Umegaki type can be found in $[10,11,12]$. Note that $\mathcal{I}_{\mathcal{A}, \mathcal{B}}^{(\mathrm{a})}(\pi) \leq \mathcal{I}_{\mathcal{A}, \mathcal{B}}^{(\mathrm{b})}(\pi)$ as it follows from Ohya and Petz [26].

The following inequality for Araki-Umegaki type can also be found in $[10,11,12]$. Similarly this inequality for Belavkin-Staszewski type holds.

Theorem 2 Let $\lambda: \mathcal{B} \rightarrow \mathcal{A}_{*}^{0}$ be an entanglement of the state $\sigma(B)=\operatorname{Tr}[\lambda(B)]$ to $\left(\mathcal{A}^{0}, \rho^{0}\right)$ with $\mathcal{A}^{0} \subseteq \mathcal{L}\left(\mathcal{G}_{0}\right), \varrho^{0}=\lambda(I)$ on $\mathcal{B}$, and $\pi=\mathrm{K}_{*} \lambda$ be entanglement to the state $\rho=\rho^{0} \mathrm{~K}$ on $\mathcal{A} \subseteq \mathcal{G}$ defined as the composition of $\lambda$ with the predual operator $\mathrm{K}_{*}: \mathcal{A}_{*}^{0} \rightarrow \mathcal{A}_{*}$ normal completely positive unital map $\mathrm{K}: \mathcal{A} \rightarrow \mathcal{A}^{0}$. Then for both mutual quantum informations, the following monotonicity holds

$$
\mathcal{I}_{\mathcal{A}, \mathcal{B}}(\pi) \leq \mathcal{I}_{\mathcal{A}^{0}, \mathcal{B}}(\lambda) .
$$


Proof: This follows from the commutativity of the following diagrams:

Applying the monotonicity property of the relative information on $\mathcal{M}=\mathcal{A} \otimes \mathcal{B}$ with respect to the predual map $\varpi_{0} \mapsto\left(\mathrm{K}_{*} \otimes \mathrm{Id}\right)\left(\varpi_{0}\right)$ corresponding to $\omega_{0} \mapsto \omega_{0}(\mathrm{~K} \otimes \mathrm{Id})$ as the ampliation $\mathrm{K} \otimes \mathrm{Id}$ of a normal completely positive unital map $\mathrm{K}: \mathcal{A} \rightarrow \mathcal{A}^{0}$.

Definition 5 The maximal quantum mutual information $\mathcal{J}_{\tilde{\mathcal{B}}, \mathcal{B}}\left(\pi_{q}\right)$ for both types as the supremum

$$
H_{\mathcal{B}}(\varsigma)=\sup _{\pi^{*}(I)=\varsigma} \mathcal{I}_{\mathcal{B}, \mathcal{A}}\left(\pi^{*}\right)=\mathcal{J}_{\mathcal{B}, \tilde{\mathcal{B}}}\left(\pi_{q}^{*}\right)
$$

over all entanglements $\pi^{*}$ of any $(\mathcal{A}, \rho)$ to $(\mathcal{B}, \sigma)$ is achieved on $\mathcal{A}^{0}=\tilde{\mathcal{B}}$, $\varrho^{0}=\tilde{\varsigma}$ by the standard quantum entanglement $\pi_{q}^{*}(A)=\varsigma^{1 / 2} \widetilde{A} \varsigma^{1 / 2}$ for a fixed $\sigma(B)=\operatorname{Tr}_{\mathcal{H}}[B \varsigma]$ is named as entangled, or true quantum entropy of each type of the state $\sigma$.

This definition for Araki-Umegaki type can be found in $[10,11,12]$.

Definition 6 We call the positive difference

$$
H_{\mathcal{B} \mid \mathcal{A}}(\pi)=H_{\mathcal{B}}(\varsigma)-\mathcal{I}_{\mathcal{A}, \mathcal{B}}(\pi)
$$

entangled (or true quantum) conditional entropy respectively of each type on $\mathcal{B}$ with respect to $\mathcal{A}$.

This definition for Araki-Umegaki type can be found in $[10,11,12]$. Obviously, the conditional mutual quantum entropies of both types are positive, unlike the "conditional entropies" considered for example in [8].

\section{$5 \quad$ Entangled Channel Capacity and its Additivity}

Entanglement-assisted quantum capacity, or entangled quantum capacity is extensively researched recently, such as entangled quantum capacity $[10,11,12]$ and entanglement-assisted quantum capacity $[20,21]$. Generally C. H. Bennett, P. W. Shor, J. A. Smolin and A. V. Thapliyal $[20,21]$ defined entanglement-assisted capacity of quantum channel via a common framework, we now discuss quantum channel capacity via entanglement via mutual quantum information entropy.

Let $\mathcal{B} \subseteq \mathcal{L}(\mathcal{H})$ be the $W^{*}$-algebra of operators in a (not necessarily finite dimensional unitary) Hilbert space $\mathcal{H}$. Generally we denote the set of states, i.e. positive unit trace operators in $\mathcal{B}(\mathcal{H})$ by $\mathcal{S}(\mathcal{H})$, the set of all $m$-dimensional projections by $\mathcal{P}_{m}(\mathcal{H})$ and the set of all projections by $\mathcal{P}(\mathcal{H})$. 
Definition 7 A quantum channel $\Lambda$ is a normal unital completely positive linear map (UCP) of $\mathcal{B}$ into the same or another algebra $\mathcal{B}^{0} \subseteq \mathcal{B}\left(\mathcal{H}^{0}\right)$. These maps admit the Kraus decomposition, which is usually written in terms of the dual map $\Lambda^{*}: \mathcal{B}_{*}^{0} \rightarrow \mathcal{B}_{*}$ as $\Lambda^{*}\left(\varsigma^{0}\right)=$ $\sum_{k} A_{k} \varsigma^{0} A_{k}^{*} \equiv \Lambda_{*}\left(\varsigma^{0}\right)$ (W. F. Stinespring [13], G. Lindblad [14], A. S. Holevo, [26]), $\Lambda(B)=\sum_{k} A_{k}^{*} B A_{k}$, for $A_{k}$ are operators $\mathcal{H}^{0} \rightarrow \mathcal{H}$ satisfying $\sum_{k} A_{k}^{*} A_{k}=I^{0}$. For example, quantum noiseless channel in the case $\mathcal{B}=\mathcal{L}(\mathcal{H}), \mathcal{B}^{0}=\mathcal{L}\left(\mathcal{H}^{0}\right)$ is described by a single isometric operator $Y: \mathcal{H}^{0} \rightarrow \mathcal{H}$ as $\Lambda(B)=Y^{*} B Y$. See for example [22,23] for the simple cases $\mathcal{B}=\mathcal{L}(\mathcal{H}), \operatorname{dim}(\mathcal{H})<\infty$.

A noisy quantum channel sends input pure states $\sigma_{0}=\rho_{0}$ on the algebra $\mathcal{B}^{0}=\mathcal{L}\left(\mathcal{H}^{0}\right)$ into mixed states described by the output densities $\varsigma=\Lambda^{*}\left(\varsigma^{0}\right)$ on $\mathcal{B} \subseteq \mathcal{L}(\mathcal{H})$ given by the predual $\Lambda_{*}=\Lambda^{*} \mid \mathcal{B}_{*}^{0}$ to the normal completely positive unital map $\Lambda: \mathcal{B} \rightarrow \mathcal{B}^{0}$ which can always be written as

$$
\Lambda(B)=\operatorname{Tr}_{\mathcal{F}_{+}}\left[Y^{\dagger} B Y\right] .
$$

Here $Y$ is a linear operator from $\mathcal{H}^{0} \otimes \mathcal{F}_{+}$to $\mathcal{H}$ with $\operatorname{Tr}_{\mathcal{F}_{+}}\left[Y^{\dagger} Y\right]=I$, and $\mathcal{F}_{+}$is a separable Hilbert space of quantum noise in the channel. Each input mixed state $\sigma^{0}$ is transmitted into an output state $\sigma=\sigma^{0} \Lambda$ given by the density operator

$$
\Lambda^{*}\left(\varsigma^{0}\right)=Y\left(\varsigma^{0} \otimes I_{+}\right) Y^{\dagger} \in \mathcal{B}_{*}
$$

for each density operator $\varsigma^{0} \in \mathcal{B}_{*}^{0}$, the identity operator $I_{+} \in \mathcal{F}_{+}$.

We follow $[10,11,12]$ to denote $\mathrm{K}_{q}$ the set of all normal transposecompletely positive maps $\kappa: \mathcal{A} \rightarrow \mathcal{B}^{0}$ with any probe algebra $\mathcal{A}$, normalized as $\operatorname{Tr} \kappa(I)=1$, and $\mathcal{K}_{q}\left(\varsigma^{0}\right)$ be the subset of $\kappa \in \mathcal{K}_{q}$ with $\kappa(I)=\varsigma^{0}$. We take the standard entanglement $\pi_{q}^{0}$ on $\left(\mathcal{B}^{0}, \sigma^{0}\right)=$ $\left(\mathcal{A}_{0}, \rho^{0}\right)$, where $\rho_{0}\left(A_{0}\right)=\operatorname{Tr}\left[A_{0} \varrho_{0}\right]$ given by the density operator $\varrho_{0}=$ $\varsigma^{0}$, and denote by $\mathrm{K}$ a normal unital completely positive map $\mathcal{A} \rightarrow$ $\mathcal{A}^{0}=\widetilde{\mathcal{A}}_{0}$ that decomposes $\kappa$ as $\kappa(A)=\varrho_{0}^{1 / 2} \widetilde{\mathrm{K}(A)} \varrho_{0}^{1 / 2}$. It defines an input entanglement $\kappa^{*}=\mathrm{K}_{*} \pi_{q}^{0}$ on the input of quantum channel as transpose-completely positive map on $\mathcal{A}_{0}=\mathcal{B}^{0}$ into $\mathcal{A}_{*}$ normalized to $\varrho=\mathrm{K}_{*} \varrho^{0}, \varrho^{0}=\widetilde{\varrho}_{0}$.

The channel $\Lambda$ transmits this input entanglement as a true-quantum encoding into the output entanglement $\pi=\mathrm{K}_{*} \pi_{q}^{0} \Lambda \equiv \mathrm{K}_{*} \lambda$ mapping $\mathcal{B}$ via the channel $\Lambda$ into $\mathcal{A}_{*}$ with $\pi(I)=\varrho$. The mutual entangled information, transmitted via the channel for quantum encoding $\kappa$ is therefore $\mathcal{J}_{\mathcal{A}, \mathcal{B}}\left(\kappa^{*} \Lambda\right)=\mathcal{J}_{\mathcal{A}, \mathcal{B}}\left(\mathrm{K}_{*} \pi_{q}^{0} \Lambda\right)=\mathcal{J}_{\mathcal{A}, \mathcal{B}}\left(\mathrm{K}_{*} \lambda\right)$, where $\lambda=\pi_{q}^{0} \Lambda$ is the standard input entanglement $\pi_{q}^{0}(B)=\varsigma_{0}^{1 / 2} \widetilde{B} \varsigma_{0}^{1 / 2}$ with $\varsigma_{0}=\widetilde{\varsigma}^{0}$, transmitted via the channel $\Lambda$. 
Lemma 4 Given a quantum channel $\Lambda: \mathcal{B} \rightarrow \mathcal{B}^{0}$, and an input state $\sigma^{0}$ on $\mathcal{B}^{0}$, the entangled input-output quantum information capacity via a channel $\Lambda: \mathcal{B} \rightarrow \mathcal{B}^{0}$ as the supremum over the set $\mathcal{K}_{q}\left(\varsigma^{0}\right)$ including true-quantum encodings $\kappa$ achieves the maximal value

$$
\mathcal{J}\left(\varsigma^{0}, \Lambda\right)=\sup _{\kappa \in \mathcal{K}_{q}\left(\varsigma^{0}\right)}\left(\kappa^{*} \Lambda\right)=\mathcal{I}_{\mathcal{A}^{0}, \mathcal{B}}(\lambda)
$$

where $\lambda=\pi_{q}^{0}$ is given by the corresponding extremal input entanglement $\pi_{q}^{0}$ mapping $\mathcal{B}^{0}=\tilde{\mathcal{A}}^{0}$ into $\mathcal{A}^{0}=\tilde{\mathcal{B}}^{0}$ with $\operatorname{Tr}\left[\pi_{q}(B)\right]=\sigma^{0}(B)$ for all $B \in \mathcal{B}^{0}$.

Note that this Lemma for Araki-Umegaki type can be found in $[10,11,12]$.

The following definition uses commutativity of diagrams:

Definition 8 Given a quantum channel $\Lambda: \mathcal{B} \rightarrow \mathcal{B}^{0}$, and a input state $\sigma^{0}$ on $\mathcal{B}^{0}$, we can define the input-output entangled information capacity as the maximal mutual quantum information

$$
\mathcal{J}\left(\varsigma^{0}, \Lambda\right)=\mathcal{I}_{\mathcal{B}^{0}, \mathcal{B}}\left(\pi_{q}^{0} \Lambda\right)
$$

for input standard entanglement of the state $\varsigma^{0}$ to the state $\varrho^{0}=\widetilde{\varsigma}^{0}$.

Note that this definition for Araki-Umegaki type can be found in $[10,11,12]$. Thus we have at least two types of such mutual quantum entropy, and obviously, $\mathcal{J}^{(\mathrm{a})}\left(\varsigma^{0}, \Lambda\right) \leq \mathcal{J}^{(\mathrm{b})}\left(\varsigma^{0}, \Lambda\right)$ with input product state $\rho_{0}^{\otimes}=\otimes_{i=1}^{n} \rho_{0}^{i}$ corresponding to the states $\rho_{0}^{i}=\sigma_{i}^{0}$ on $\mathcal{B}_{i}^{0}$.

Here and below for notational simplicity we implement the agreements $\mathcal{A}_{0}^{i}=\mathcal{B}_{i}^{0}, \rho_{0}^{i}=\sigma_{i}^{0}, \mathcal{A}_{0}^{\otimes}=\otimes_{i=1}^{n} \mathcal{B}_{i}^{0}, \rho_{0}^{\otimes}=\otimes_{i=1}^{n} \sigma_{i}^{0}$ such that $\varsigma_{0}^{\otimes}=\otimes_{i=1}^{n} \varrho_{i}^{0}$ is transposed input state $\widetilde{\varrho}_{0}^{\otimes}=\otimes_{i=1}^{n} \widetilde{\varsigma}_{i}^{0}$ on $\mathcal{B}_{0}^{\otimes}=\otimes_{i=1}^{n} \mathcal{A}_{i}^{0}$ with $\widetilde{\mathcal{B}}_{i}^{0}=\mathcal{A}_{i}^{0} \equiv \mathcal{B}_{0}^{i}=\widetilde{\mathcal{A}}_{0}^{i}, \widetilde{\varsigma}_{i}^{0}=\varrho_{i}^{0} \equiv \varsigma_{0}^{i}=\widetilde{\varrho}_{0}^{i}$.

Let $\Lambda_{i}$ be channels respectively from the algebra $\mathcal{B}_{i}$ on $\mathcal{H}_{i}$ to $\mathcal{B}_{i}^{0}$ on $\mathcal{H}_{i}^{0}$ for $i=1,2, \ldots, n$, and let $\Lambda^{\otimes}=\otimes_{i=1}^{n} \Lambda_{i}$ be their tensor product.

We now show the additivity property of this entangled inputoutput quantum information capcity under a given input state, using monotonicity property (as indicated in $[10,11,12]$ for Araki-Umegaki type).

Theorem 3 Let $\Lambda^{\otimes}$ be product channel from the algebra $\mathcal{B}^{\otimes}=$ $\otimes_{i=1}^{n} \mathcal{B}_{i}$ to $\mathcal{A}_{0}^{\otimes}=\otimes_{i=1}^{n} \mathcal{A}_{0}^{i}$, and let $\rho_{0}^{\otimes}=\otimes_{i=1}^{n} \rho_{0}^{i}$ be the tensor product of input states $\sigma_{0}^{i}$ on $\mathcal{B}_{0}^{i}$, then

$$
\mathcal{J}\left(\varrho_{0}^{\otimes}, \Lambda^{\otimes}\right)=\sum_{i=1}^{n} \mathcal{J}\left(\varrho_{0}^{i}, \Lambda_{i}\right) .
$$

Proof: Take $\Lambda_{i *}: \mathcal{B}_{i *}^{0} \rightarrow \mathcal{B}_{i *}$, and $\varrho_{0}^{i} \in \mathcal{B}_{i *}^{0}, \varsigma_{i}=\Lambda_{i *}\left(\varrho_{0}^{i}\right) \in \mathcal{B}_{i *}$, and $\mathrm{K}_{*}^{(n)}: \mathcal{A}_{*}^{\otimes} \rightarrow \mathcal{A}_{*}^{(n)}$, where $\mathcal{A}_{0 *}^{\otimes}=\otimes_{i=1}^{n} \mathcal{B}_{i *}^{0}$, but $\mathcal{A}_{*}^{(n)}$ is predual 
to a general, not necessarily product algebra $\mathcal{A}^{(n)} \subseteq \mathcal{L}\left(\mathcal{G}^{(n)}\right)$. For $\pi^{(n)}=\mathrm{K}_{*}^{(n)} \pi_{q}^{0 \otimes} \Lambda^{\otimes}$, below we consider quantum mutual information $\mathcal{I}_{\mathcal{A}^{(n)}, \mathcal{B} \otimes}\left(\pi^{(n)}\right)$ as relative quantum entropy

$$
\mathcal{R}\left(\left(\mathrm{K}_{*}^{(n)} \otimes \Lambda_{*}^{\otimes}\right) \widetilde{\varpi}_{0}^{\otimes}: \mathrm{K}_{*}^{(n)}\left(\varsigma_{0}^{\otimes}\right) \otimes \Lambda_{*}^{\otimes}\left(\varrho_{0}^{\otimes}\right)\right),
$$

where $\widetilde{\varpi}_{0}^{\otimes}=\otimes_{i=1}^{n} \widetilde{\varpi}_{0}^{i}$ is the density operator of the standard compound state $\otimes_{i=1}^{n} \omega_{0}^{i}$ with $\omega_{0}^{i}\left(A_{i} \otimes B_{i}\right)=\varpi_{i}^{0}\left(A_{i} \otimes B_{i}\right)=\operatorname{Tr}\left[B_{i} \sqrt{\varrho_{i}^{0}} \widetilde{A}_{i} \sqrt{\varrho_{i}^{0}}\right]$ for $A_{i} \in \widetilde{\mathcal{B}}_{i}^{0}, B_{i} \in \mathcal{B}_{i}^{0}$, corresponding to $\varsigma_{i}^{0}=\varrho_{0}^{i}$.

Applying monotonicity property (Lemma 3) of quantum relative entropy to the probe system $\left(\mathcal{G}^{(n)}, \mathcal{A}^{(n)}\right)$ for this given $\varrho_{0}^{i}$ and $\Lambda_{i}$, we obtain

$$
\begin{aligned}
& \mathcal{R}\left(\left(\mathrm{K}_{*}^{(n)} \otimes \Lambda_{*}^{\otimes}\right) \widetilde{\varpi}_{0}^{\otimes}: \mathrm{K}_{*}^{(n)}\left(\varsigma_{0}^{\otimes}\right) \otimes \Lambda_{*}^{\otimes}\left(\varrho_{0}^{\otimes}\right)\right) \\
\leq & \mathcal{R}\left(\left(\mathrm{Id}^{\otimes} \otimes \Lambda^{\otimes}\right) \widetilde{\varpi}_{0}^{\otimes}: \operatorname{Id}^{\otimes}\left(\varsigma_{0}^{\otimes}\right) \otimes \Lambda_{*}^{\otimes}\left(\varrho_{0}^{\otimes}\right)\right) \\
= & \sum_{i=1}^{n} \mathcal{R}\left(\left(\operatorname{Id} \otimes \Lambda_{i *}\right)\left(\widetilde{\varpi}_{0}\right): \operatorname{Id}\left(\varsigma_{0}^{i}\right) \otimes \Lambda_{i *}\left(\varrho_{0}^{i}\right),\right.
\end{aligned}
$$

where $\varsigma_{0}^{i}=\varrho_{i}^{0}=\widetilde{\varrho}_{0}^{i}, \varrho_{0}^{i}=\varsigma_{i}^{0}=\widetilde{\varsigma}_{0}^{i}$.

The suprema over $\mathrm{K}^{(n)}$ is achieved on $\mathrm{K}^{(n)}=\mathrm{Id}^{\otimes}$ identically mapping $\mathcal{A}^{(n)}=\otimes_{i=1}^{n} \mathcal{A}_{0}^{i}$ to $\mathcal{B}_{0 *}^{\otimes}=\otimes_{i=1}^{n} \mathcal{B}_{0}^{i}$, where $\mathcal{B}_{0}^{i}=\widetilde{\mathcal{B}}_{i}^{0}$, coinciding with such $\mathcal{A}^{(n)}$ due to $\mathcal{A}_{0}^{i}=\widetilde{\mathcal{B}}_{i}^{0}$.

Thus $\mathcal{J}\left(\varrho_{0}^{\otimes}, \Lambda^{\otimes}\right)=\sum_{i=1}^{n} \mathcal{J}\left(\varrho_{0}^{i}, \Lambda_{i}\right)$.

Definition 9 Given a normal unital completely positive map $\Lambda$ : $\mathcal{B} \rightarrow \mathcal{A}$, the suprema

$$
C_{q}(\Lambda)=\sup _{\kappa \in \mathcal{K}_{q}} \mathcal{I}_{\mathcal{A}, \mathcal{B}}\left(\kappa^{*} \Lambda\right)=\sup _{\varsigma^{0}} \mathcal{J}\left(\varsigma^{0}, \Lambda\right)
$$

is called the quantum channel capacity via entanglement, or q-capacity.

Note that this definition for Araki-Umegaki type can be found in $[10,11,12]$, there we have two types of entangled channel capacities, and obviously $C_{q}^{(\mathrm{a})}(\Lambda) \leq C_{q}^{(\mathrm{b})}(\Lambda)$.

Lemma 5 Let $\Lambda(B)=Y^{\dagger} B Y$ be a unital completely positive map $\Lambda: \mathcal{B} \rightarrow \mathcal{B}^{0}$ describing a quantum deterministic channel by an isometry $Y: \mathcal{H}^{0} \rightarrow \mathcal{H}$. Then

$$
\begin{gathered}
\mathcal{J}\left(\varsigma^{0}, \Lambda\right)=H_{\mathcal{B}_{0}}\left(\varsigma^{0}\right), \\
C_{q}(\Lambda)=\ln \operatorname{dim} \mathcal{B}^{0} .
\end{gathered}
$$

Note that this Lemma for Araki-Umegaki type can be found in $[10,11,12]$. 
Let $\Lambda^{\otimes}$ be product channel from the algebra $\mathcal{B}^{\otimes}=\otimes_{i=1}^{n} \mathcal{B}_{i}$ to $\mathcal{A}_{0}^{\otimes}=\otimes_{i=1}^{n} \mathcal{B}_{i}^{0}$. The additivity problem for quantum channel capacity via entanglement is if it is true that

$$
\mathcal{C}_{q}\left(\Lambda^{\otimes}\right)=\sum_{i=1}^{n} \mathcal{C}_{q}\left(\Lambda_{i}\right)
$$

We now still follow the idea of $[10,11,12]$ to give a proof of this additivity property via operational approach using monotonicity property (as indicated in $[10,11,12]$ for Araki-Umegaki type).

Theorem 4 Let $\Lambda^{\otimes}$ be product channel from the algebra $\mathcal{B}^{\otimes}=$ $\otimes_{i=1}^{n} \mathcal{B}_{i}$ to $\mathcal{A}_{0}^{\otimes}=\otimes_{i=1}^{n} \mathcal{B}_{i}^{0}$, then

$$
\mathcal{C}_{q}\left(\Lambda^{\otimes}\right)=\sum_{i=1}^{n} \mathcal{C}_{q}\left(\Lambda_{i}\right)
$$

Proof: It simply follows from the additivity (31). Indeed,

$$
C_{q}\left(\Lambda^{\otimes}\right)=\sup _{\kappa \in \mathcal{K}_{q}^{(n)}} \mathcal{I}_{\mathcal{A}^{(n), \mathcal{B}}}\left(\kappa^{*} \Lambda^{\otimes}\right)=\sup _{\varrho_{0}^{\otimes}} \mathcal{J}\left(\varrho_{0}^{\otimes}, \Lambda^{\otimes}\right)=\sup _{\varrho_{0}^{\otimes}} \sum_{i=1}^{n} \mathcal{J}\left(\varrho_{0}^{i}, \Lambda_{i}\right)
$$

Therefore by further taking suprema over $\varrho_{0}^{\otimes}$ as over independently for each $i=1,2, \ldots, n$, thus we have

$$
\mathcal{C}_{q}\left(\Lambda^{\otimes}\right)=\sum_{i=1}^{n} \sup _{\varrho_{0}^{\otimes}} \mathcal{J}\left(\varrho_{0}^{i}, \Lambda_{i}\right)=\sum_{i=1}^{n} \mathcal{C}_{q}\left(\Lambda_{i}\right)
$$

which is the additivity property of entangled quantum channel capacity due to encodings via entanglement obviously.

Remark 4 Note that there is no such additivity for the Holevo capacity for a arbitrary channel $\Lambda: \mathcal{B} \rightarrow \mathcal{B}^{0}$. Indeed, this smaller, semiclassical capacity is defined as the supremum

$$
C_{d}(\Lambda)=\sup _{\kappa \in \mathcal{K}_{d}} \mathcal{I}_{\mathcal{A}, \mathcal{B}}\left(\kappa^{*} \Lambda\right)
$$

over the smaller class $\mathcal{K}_{d} \subseteq \mathcal{K}_{q}$ of the diagonal [10-12] (semiclassical) encodings $\kappa: \mathcal{A} \rightarrow \mathcal{B}_{*}^{0}$ corresponding to only diagonal (Abelian) algebras $\mathcal{A}$. This supremum cannot in general be achieved on the standard entanglement of $\mathcal{A}^{0}=\widetilde{\mathcal{B}}^{0} \equiv \mathcal{B}_{0}$ if $\mathcal{A}^{0}$ is non Abelian corresponding to the non Abelian input algebra $\mathcal{B}^{0}$. Therefore the supremum $\mathcal{C}_{d}\left(\Lambda^{\otimes}\right) \leq \sum_{i=1}^{n} \mathcal{C}_{d}\left(\Lambda_{i}\right)$ can be achieved not on a product Abelian algebra $\mathcal{A}^{(n)}$ as is was in the true quantum case where we could take $\mathcal{A}^{(n)}=\otimes_{i=1}^{n} \mathcal{B}_{0}^{i}$ with non Abelian $\mathcal{B}_{0}^{i}=\widetilde{\mathcal{B}}_{i}^{0}$. 


\section{Conclusion}

So far, continuing in this paper research on quantum channel capacity for one-way communication via entanglement following $[10,11,12,29]$, we treated two types of quantum mutual information via entanglement in algebraic approach and corresponding quantum channel capacities via entanglement in operational approach. Using monotonicity property of quantum mutual information of a- and b-type introduced in $[10,29]$ we proved additivity property of quantum channel capacities via entanglement, therefore extending the results of V. P. Belavkin $[10,10 \mathrm{a}]$ to products of arbitrary quantum channel to quantum relative entropy of both Araki-Umegaki type and Belavkin-Staszewski type.

As written in the introduction, quantum channel capacities can have several different formulations when considering to send classical information or quantum information, one-way or two-way communication, prior or via entanglement, etc. in the form of different constraints on the encoding class $\mathcal{K}$. Anyway general quantum channel capacity with different constraints is still a big open and challenging research problem in quantum information theory. Much more open problems can be found in [9]. There we anticipate some research on quantum channel capacity for two-way communication and prior or via entanglement, i.e. trading communication and entanglement for quantum channel capacity.

Another natural problem in this direction is to compare true quantum capacities in quantity for some interesting quantum channels with other smaller capacities under constraints, such as Holevo capacity, entanglement-assistant capacity, etc., and find for which channels they coincide.

The third natural problem in this direction is to consider quantum mutual information via entanglement and corresponding quantum channel capacities via entanglement for $\gamma$ type since [29] studied this third and more general quantum relative entropy in quantum information, which also meet more natural axiomatic properties of relative entropy.

Tracing through the original research ideas on quantum mutual information via entanglement and corresponding quantum channel capacities via entanglement, it is easy to find that the maximal quantum information and capacity is achieved on maximal (standard) entanglement for a given state and on the absolutely maximal entanglement (which exists only in finite dimensions) without constraint on the input state.

Generally how to access those capacities, using physically imple- 
mentable operations for encodings and decodings, such as in this direction of quantum channel capacity for one-way communication via entanglement, is of course an open problem in quantum information and quantum computation.

All those problems wait forthcoming papers in the future.

\section{$7 \quad$ References}

[1] C. H. Bennett, G. Brassard, C. Crepeau, R. Jozsa, A. Peres and W. K. Wootters, Teleporting an unknown quantum state via dual classic and Einstein-Podolsky-Rosen channels, Phys. Rev. Lett. 70(1993)18951899.

[2] A. Ekert, Quantum cryptography based on Bell's Theorem, Phys. Rev. Lett. 67(1990)661-663.

[3] R. Jozsa and B. Schumacher, A new proof of the quantum noiseless coding theorem, J. Mod. Opt. 41(1994)2343-2350.

[4] H. Araki, Relative Entropy of states of von Neumann Algebras, Publications RIMS, Kyoto University, 11,809(1976)

[5] H. Umegaki, Kodai Math. Sem. Rep. 14,59 (1962)

[6] L. D. Landau, and E. M. Lifschitz, Quantum Mechanics (NonRelativistic Theory), 3rd ed. Oxford, England: Pergamon Press, 1977.

[7] J. von Neumann, Mathematical Foundations of Quantum Mechanics,Princeton University Press, 1955.

[8] Michael A. Nielsen and Isaac L. Chuang, Quantum Computation and Quantum Information, Cambridge University Press 2000.

[9] Peter Shor, Quantum Information Theory: Results and Open Problems, Geom. Funct. Anal., Special Volume-GAFA2000,816-838(2000).

[10] V. P. Belavkin, On Entangled Quantum Capacity. In: Quantum Communication, Computing, and Measurement 3. Kluwer/Plenum, 2001, 325-333.

[10a] V. P. Belavkin, On Entangled Information and Quantum Capacity, Open Sys. and Information Dyn, 8:1-18, 2001.

[11] V. P. Belavkin, M. Ohya, Quantum Entropy and Information in Discrete Entangled States, Infinite Dimensional Analysis, Quantum Probability and Related Topics 4 (2001) No. 2, 137-160.

[12] V. P. Belavkin, M. Ohya, Entanglement, Quantum Entropy and Mutual Information, Proc. R. Soc. Lond. A 458 (2002) No. 2, $209-231$

[13] W. F. Stinespring, Proc. Amer. Math. Soc. 6, p.211(1955)

[14] G. Lindblad, Entropy, Information and Quantum Measurements, Comm. in Math. Phys. 33, p.305-322(1973)

[15] Peter Levay, The geometry of entanglement: metrics, connections and the geometric phase, J. Phys. A37 (2004) 1821-1842; 
quant-ph/0306115.

[16] R. Penrose, Report in NCG workshop in Newton Institute, Cambridge, Sept., 2006.

[17] Peter Levay, The twistor geometry of three-qubit entanglement, quant-ph/0403060.

[18] V. P. Belavkin, R. L. Stratonovich, Optimization of Quantum Information Processing Maximizing Mutual Information,Radio Eng. Electron. Phys., 19 (9), p. 1349, 1973; quant-ph/0511042.

[19] Benjamin Schumacher, and Michael D. Westmorel, Quantum mutual information and the one-time pad, quant-ph/0604207.

[20] C. H. Bennett, P. W. Shor, J. A. Smolin and A. V. Thapliyal, Entanglement assisted classical capacity of noisy quantum channels, Phys. Rev. Lett., vol. 83, pp. 3081-3084, 1999.

[21] C. H. Bennett, P. W. Shor, J. A. Smolin and A. V. Thapliyal, Entanglement-Assisted Capacity of a Quantum Channel and the Reverse Shannon Theorem, quant-ph/0106052.

[22] A. S. Holevo, Quantum coding theorems, Russian Math. Surveys 53:6, 1295-1331, 1998; quant-ph/9808023.

[23] G. Lindblad, Quantum entropy and quantum measurements, in: Proc. Int. Conf. on Quantum Communication and Measurement, ed. by C. Benjaballah, O. Hirota, S. Reynaud, Lect. Notes Phys.378, 71-80, Springer-Verlag, Berlin 1991.

[24] N. Cerf and G. Adami, Von Neumann capacity of noisy quantum channels, Phys. Rev. A 56, pp3470-3483(1997).

[25] V. P. Belavkin and P. Staszewski, C*-algebraic generalization of relative entropy and entropy, Ann. Inst. Henri Poincare, 37, Sec. A, 51-58, 1982.

[26] M. Ohya and D. Petz, Quantum Entropy and its Use, SpringerVerlag, Berlin, 1993.

[27] V. P. Belavkin, Quantum stochastic positive evolutions: characterization, construction, dilation, Commun. Math. phys. 184 (1997) 533-566.

[28] A. Einstein, B. Podolsky, and N. Rosen, Can Quantum-Mechanical Description of Physical Reality Be Considered Complete? Phys. Rev. 47, 777-780, 1935.

[29] S. J. Hammersley and V. P. Belavkin, Information Divergence for Quantum Channels, Infinite Dimensional Analysis.In: Quantum Information and Computing. World Scientific, Quantum Probability and White Noise Analysis,VXIX (2006) 149-166.

[30] B. Schumacher, Sending entanglement through noisy quantum channels, Phys. Rev. A 54, pp 2614-2628, 1996. 